\title{
Folding intraocular lenses: materials and methods
}

The change in cataract surgery technique to ever smaller incisions requires the use of folding intraocular lenses (IOLs). Leaming ${ }^{1}$ in his annual survey of practice styles of members of the American Society of Cataract and Refractive Surgeons reports that in $199538 \%$ of respondents were using a $3 \mathrm{~mm}$ incision, with $14 \%$ using silicone and 9\% acrylic IOLs, a considerable change from 1994. Polymethylmethacrylate (PMMA) remains the gold standard IOL material since first implanted by Ridley ${ }^{2}$ in 1949 with a track record of close to 50 years, and it still is the preferred material of US surgeons in $1995 .{ }^{1}$

Which materials are in use in folding IOLs in 1997 and what methods of implantation are available? Silicone has been in use as an IOL material since the early 1980s with Food and Drugs Administration (FDA) approval obtained in 1990 for a three piece silicone lens. Silicone in IOLs is a biologically inert polymer, polydimethylsiloxane (PDMS), which cannot leach out - unlike silicone in breast implants. The earlier lenses had a refractive index of 1.41 making folding of three piece lenses over $22 \mathrm{D}$ difficult. The refractive index of most three piece silicone IOLs is now 1.47 reducing optical thickness and facilitating folding. Plate haptic silicone lenses have a refractive index of 1.41 making a thicker lens which, the manufacturers suggest, fills the capsular bag reducing the incidence of posterior capsular opacification. Silicone folds easily but springs open unless delivery is controlled. It is difficult to handle when wet as it becomes slippery. Silicone IOLs should not be used in the presence of silicone oil in the vitreous cavity, or if silicone oil may be required in the future, as condensations will occur on the lens, in particular in the presence of a posterior capsulotomy. ${ }^{3}$ Silicone lenses are currently the most popular folding IOLs ${ }^{1}$ with two styles in use-a three piece design with a silicone optic and polypropylene or PMMA haptics and a one piece plate haptic lens with an overall length of $10.50 \mathrm{~mm}$.

Acrylic as a lens material is relatively new with FDA approval obtained in December 1994 and an increase in use in the USA from $2 \%$ to $9 \%$ between 1994 and $1995 .{ }^{1}$ Acrylics are polymers synthesised from esters of acrylic or methacrylic acid. Acrylic IOLs have a refractive index of 1.55 and the hardness of the acrylic is temperature dependent. The high refractive index gives acrylic lenses the lowest edge thickness of all available lens materials. At low temperatures the lens feels almost like PMMA and folding is facilitated by warming the lens. Acrylic lenses fold and unfold slowly and can be handled when wet. If the lens is too warm it can become sticky and unfolding can be difficult. Condensations occur less frequently on acrylic lenses than PMMA and silicone lenses following fluid-air exchange. ${ }^{3}$ Present lens design is three piece, with an acrylic optic of 5.5 or $6.0 \mathrm{~mm}$ diameter and PMMA haptics. A one piece acrylic lens is undergoing clinical trials.

Hydrogel, a soft hydrophilic material developed for biomedical use has a long history of use as a biomedical material $^{4}$ and is now used in folding IOLs. The material used is polyhydroxyethylmethacrylate (PolyHema) with a water content varying from $18 \%$ to $30 \%$ and a refractive index of 1.47. Hydrogel lenses fold and unfold faster than acrylic and are more controllable than silicone. Because of their water content they must be kept hydrated until implantation, making lens presentation in the operating theatre slightly difficult. Hydrogel lenses are available with a hydrogel optic of $6 \mathrm{~mm}$ bonded to PMMA optics and a single piece lens which is currently in clinical trials.

Surgical results on the use of folding lens implantation are available, silicone having the longest track record with millions of silicone lenses implanted worldwide and many published results. Cumming ${ }^{5}$ in 1993 compared the results of 503 patients implanted with plate haptic silicone lenses with 253 patients implanted with three piece silicone lenses. Visual results in both groups were excellent with $97.5 \%$ of patients with plate haptic lenses seeing $20 / 40$ or better but he found a higher incidence of cystoid macular oedema with a visual acuity of less than $20 / 40$ in the three piece group than in the plate haptic group, $2.4 \%$ versus $0 \%$. Complications were reported in $10.3 \%$ of eyes implanted with a three piece lens compared with $3.2 \%$ of eyes implanted with the plate haptic lens. The three piece lens in this study did have prolene loops which are known to have a higher complication rate.

Results of acrylic lens implantation have been published by Oshika et al ${ }^{6}$ who found that $96.9 \%$ of patients had a corrected visual acuity of $20 / 40$ or better at day one and at 2 years postoperatively $100 \%$ had $20 / 40$ or better and $86.3 \% 20 / 20$ or better. Flare intensity measured with the laser flare/cell meter was less than with other types of IOLs measured and no other postoperative complications were encountered.

Hydrogel one piece lenses ${ }^{7}$ have produced good visual results, with Percivals and Jafree reporting $100 \%$ of patients seeing $6 / 9$ or better in 1994 ; the only adverse reaction reported was asymptomatic decentration in two cases. The author has obtained good results from clinical trials on a hydrogel lens with PMMA haptics with $97.1 \%$ of eyes seeing $20 / 40$ or better at $12-14$ months postoperatively and no sight threatening complications.

What effect do these new materials have on posterior capsular opacification and YAG capsulotomy rates? Silicone plate haptic lenses have been reported as having a lower YAG laser capsulotomy rate than three piece silicone lenses, ${ }^{5} 2.4 \%$ versus $7.1 \%$, with the YAG laser capsulotomy also being necessary at an earlier stage with the three piece lens. Posterior capsular opacification has been reported to be extremely low with acrylic lenses but Oshika et al reported a YAG laser capsulotomy rate of $11.1 \%$ (seven eyes of 64 patients) implanted. ${ }^{6}$ Hydrogel lenses in their current form have only been implanted since 1993 and no published results are available on YAG capsulotomy rates. Exposure to YAG laser of silicone, acrylic, and hydrogel lenses shows equivalent or less damage than PMMA but plate haptic silicone lenses should not undergo early YAG capsulotomy owing to the risk of lens dislocation into the vitreous cavity.

Cellular proliferation on an IOL surface has been reported as a good indicator of biocompatibility of the lens material and postoperative inflammatory reaction with hydrophilic surfaces showing a lower percentage of cells compared with hydrophobic surfaces. ${ }^{9}$ A low percentage of cells has been found on hydrogel lenses and it has been proposed that hydrophilic surfaces are more biocompatible. ${ }^{4}$ The author has seen lens epithelial cell 
migration from the anterior capsulorhexis edge on hydrogel IOLs but these have not been visually threatening and have been noted previously by Apple and Blotnik ${ }^{10}$ with a small capsulorhexis. Foreign body giant cells were found more often on silicone disc IOLs than on three piece silicone IOLs. ${ }^{9}$ Amon and Menapeace attribute this to traumatisation of ocular tissues during implantation. Acrylic lenses show a very low rate of cellular adhesion (D Spalton, personal communication) and this has not yet been fully explained.

Implantation techniques continue to improve with a wide variety of folding techniques and instruments and considerable debate continues about the optimal incision size for each lens. ${ }^{11}$ Plate haptic silicone lenses can be placed in a loading deck in an inserter system and, using a viscoelastic material, the lens can be injected into the capsular bag through a $3 \mathrm{~mm}$ incision. However, to ensure a non-stretched incision, the incision size should equal the size of the lens perimeter divided by two. If the incision is not sufficiently large, a fish mouth effect with tearing will occur. Steinert and Deacon ${ }^{11}$ have shown that the phacoemulsification incision enlarges at each separate procedure during surgery and suggests that clinical studies which assume that the initial incision size equals the final incision size may be erroneous. They also say that in the absence of actual measurement of an incision, the surgeon might believe that a foldable lens could be inserted through a 2.8 $\mathrm{mm}$ incision. However, their measurements show that the actual range of the final incision size was $3.2-3.5 \mathrm{~mm}$. They believe that 'corneal elasticity' which many surgeons believe allows them to insert a larger lens through a smaller incision does not occur and their work shows that tearing of the incision results. Three piece lenses are folded manually and then grasped with the folding forceps and inserted into the eye through a $3.5 \mathrm{~mm}$ or larger incision.
In 1997 we have the choice not only of the many different types of intraocular lenses, but of intraocular lenses made of four different materials. We are still seeking the answers to which is the most biocompatible material with the lowest rate of posterior capsular opacification and lowest complication rate. We are also continuing to seek the implantation technique which is least traumatic both to the patient's eye and the intraocular lens. By working together on clinical trials and sharing our results we will continue the work on lens implantation begun by Ridley in the UK in 1949.

H C SEWARD

Croydon Eye Unit, 33 Mayday Road,

Thornton Heath, Surrey CR7 7YE

1 Leaming D. Practice styles and preferences of ASCRS members: 1995 survey. 7 Cataract Refract Surg 1996;22:931-9.

2 Ridley H. Further observations on intraocular acrylic lenses in cataract surgery. Am f Ophthalmol 1953;36:98-106.

3 Hainsworth DP, Chen SI, Cox TA, Jaffe GJ. Condensation on polymethylmethacrylate, acrylic polymer, and silicone intraocular lenses after fluid-air exchange in rabbits. Ophthalmology 1996;103:1410-8.

4 Barrett GD. The evolution of implants. In: Sourdille P, ed. Evolution of microsurgery 1991;22:70-1.

5 Cumming JS. Postoperative complications and uncorrected acuities after implantation of plate haptic silicone and three-piece silicone IOLs. 7 Cataract Refract Surg 1993;19:263-75.

6 Oshika T, Suzuki Y, Kizaki H, Yaguchi S. Two year clinical study of a soft acrylic intraocular lens. F Cataract Refract Surg 1996;22:104-9.

7 Percivals PB, Jafree AJ. Preliminary results with a new hydrogel intraocular lens. Eye 1994;8:672-5.

8 Barrett GD. A new hydrogel intraocular lens design. $\mathcal{F}$ Cataract Refract Surg 1994;20:18-25.

9 Amon M, Menapeace R. In vivo documentation of cellular reactions on lens surfaces for assessing the biocompatibility of different intraocular implants. Eye 1994;8:649-56.

10 Apple DJ, Blotnik C. Post-operative lens deposits (letter). $\mathcal{F}$ Cataract Refract Surg 1993;19:441.

11 Steinert RF, Deacon J. Enlargement of incision width during phacoemulsification and folded intraocular lens implant surgery. Ophthalmology 1996;3: $220-5$. 